\title{
Transverse Cervical Artery Flap Repair of Benign Acquired Tracheoesophageal Fistula
}

\author{
Jourdain D. Artz, MD ${ }^{1}$ Daniel Yoo, MS² Juan José Gilbert-Fernández, MD ${ }^{1}$ Rohan R. Walvekar, MD \\ William H. Risher, MD ${ }^{4}$ Charles Dupin, MD $^{1}$
}

\footnotetext{
1 Division of Plastic and Reconstructive Surgery, Department of Surgery, Louisiana State University Health Science Center, New Orleans, Louisiana

${ }^{2}$ Louisiana State University Health Science Center, New Orleans, Louisiana

3 Department of Otolaryngology Head and Neck Surgery, Louisiana

State University Health Science Center, New Orleans, Louisiana

${ }^{4}$ Division of Cardiothoracic Surgery, Department of Surgery, Louisiana

State University Health Science Center, New Orleans, Louisiana
}

\begin{abstract}
Address for correspondence Daniel Yoo, MS, Louisiana State University Health Science Center, New Orleans, LA 70006 (e-mail: dyoo@Isuhsc.edu; danielyoo08@gmail.com).
\end{abstract}

J Reconstr Microsurg Open 2019;4:e9-e13.

\begin{abstract}
Keywords

- tracheoesophageal fistula

- transverse cervical artery flap

- repair

Acquired tracheoesophageal fistulas are rare but associated with significant morbidity and mortality. The majority of cases are due to prolonged or complicated endotracheal intubation, tracheostomy, or esophageal malignancy, or subsequent to radiation or chemotherapy for treatment of the latter. Other etiologies include esophageal stenting and complications secondary to endoscopic procedures. The pathophysiology involves chronic inflammation of the esophagus or posterior wall of the trachea, ultimately promoting fistulization between these two structures. Risk factors primarily depend on the etiology; however, excessive balloon pressures and prolonged intubation are among the strongest predictors of acquired tracheoesophageal fistula. In two reported cases, intubation with persistent air leaks resulted in fistulization. Patients present with refractory pneumonia, aspiration, hypoxemia, acute respiratory distress, enteral feed in endotracheal aspirate, or gastric distention following extubation. It can be difficult to distinguish normal functional deterioration from deterioration secondary to intubation. Up to $51 \%$ of patients intubated for at least 48 hours may experience dysphagia following extubation. Ultimately, the diagnostic algorithm includes an esophagogram, followed by imaging with computed tomography (CT) scan, and, more recently, CT scan with three-dimensional reconstructions, a bronchoscopy, and an esophagoscopy. Spontaneous closure rarely occurs, and the primary treatment modalities include interventional therapy with stenting via bronchoscopy, esophagoscopy, or surgical correction. Surgical intervention is associated with higher risks due to surrounding vital anatomy and, often, technical challenges requiring multispecialty care. Our case study presents a novel and effective method of repairing a benign acquired tracheoesophageal fistula utilizing the transverse cervical artery flap.
\end{abstract}

Acquired tracheoesophageal fistulas (TEF) are rare $(\leq 1 \%$ of patients) but are associated with significant morbidity and high mortality if untreated. ${ }^{1,2}$ The majority of cases are due to prolonged or complicated endotracheal intubation (EI), tracheostomy, or esophageal malignancy, or subsequent to radiation or chemotherapy for treatment of the latter. Other etiologies include esophageal stenting and complications secondary to endoscopic procedures. ${ }^{2-6}$ The pathophysiology is thought to involve chronic inflammation of the esophagus or posterior wall of the trachea, ultimately received

September 5, 2018 accepted after revision November 27, 2018
DOI https://doi.org/

10.1055/s-0039-1678576. ISSN 2377-0813.
Copyright $\odot 2019$ by Thieme Medical Publishers, Inc., 333 Seventh Avenue, New York, NY 10001, USA. Tel: +1(212) 584-4662.
License terms

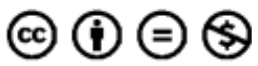


promoting a fistula between these two structures. ${ }^{1,6-8}$ According to literature, risk factors primarily depend on the etiology; however, excessive balloon pressures, defined by greater than $20 \mathrm{~mm} \mathrm{Hg}$, and prolonged intubation, defined as longer than 2 weeks, are among the strongest predictors of acquired TEF. ${ }^{1,7}$ In two reported cases, persistent air leaks (i.e., requiring reinflation or increased balloon volume) resulted in TEF. ${ }^{7}$

Patients commonly present with refractory pneumonia, aspiration, hypoxemia, acute respiratory distress, enteral feed in endotracheal aspirate, or gastric distention following extubation. ${ }^{2}$ In some cases, it can be difficult to distinguish normal functional deterioration from deterioration that is secondary to intubation; for example, up to $51 \%$ of patients intubated for at least 48 hours may experience dysphagia following extubation. ${ }^{9}$ The diagnostic algorithm includes an esophagogram, followed by imaging with a CT scan, and, more recently, a CT scan with three-dimensional reconstructions, a bronchoscopy, and an esophagoscopy. ${ }^{10}$

Spontaneous closure rarely occurs, and the primary treatment modalities include interventional therapy with stenting via bronchoscopy, esophagoscopy, or surgical correction, which is the most definitive. However, surgical intervention is associated with higher risks due to the involvement of vital anatomy and, often, technical challenges requiring multispecialty care for surgical access and repair. Mortality rates of $5.7 \%$ and complications rates of $>50 \%$ following surgical intervention have been reported. ${ }^{9,11}$ In addition, adjunct supportive management is commonly utilized; this type of management may entail enteral and parenteral alimentation modalities, including gastric tubes, antibiotics, elimination of airway secretions, and intravenous hyperalimentation. ${ }^{2}$

\section{Case Description}

A 69-year-old male patient presented with a past medical history of chronic obstructive pulmonary disease. He presented to our hospital after initially suffering from cardiac arrest while on a cruise ship. Advanced cardiac life support was initiated, and he received three rounds of cardiopulmonary resuscitation, two shocks from the automated external defibrillator, and two boluses of epinephrine before return of spontaneous circulation. He underwent several attempts for endotracheal intubation in the field, which were eventually successful in securing an airway. He was brought to University Medical Center New Orleans where he was found to have suffered from a myocardial infarction with subsequent ventricular fibrillation. He was admitted to the hospital for critical care management and coronary stenting.

The patient showed improvement; however, after failing a spontaneous breathing trial, he was reintubated with intermittent positive pressure ventilation. His sputum cultures were also positive for Haemophilus parainfluenzae for which he was started on a course of cefotaxime. Over the course of his stay, his endotracheal tube was replaced due to malfunction, his secretions worsened, and the patient eventually became septic requiring the use of norepinephrine and vasopressin. Subsequent bronchial alveolar lavage cultures also grew gram-positive cocci; he was then started on vancomycin. Eventually, his condition stabilized. Despite decreased secretions and broad-spectrum antibiotic treatment, his respiratory status remained compromised due to generalized weakness, high-pressure support requirements, and delirium/agitation. His condition gradually improved and on hospital day 11, he was extubated to nasal intermittent positive pressure ventilation. The patient initially tolerated the transition; however, he rapidly decompensated the next morning and required emergent intubation due to hypoxemia. On further exam, copious residual tube feed was found in his upper airway and oropharynx, causing obstruction. Bronchial alveolar lavage and tracheostomy were planned for true ventilator weaning. He was also restarted on vancomycin and cefepime given his aspiration of gastric contents. During this time, he suffered from another episode of cardiac arrest in the setting of hypoxemia likely secondary to airway obstruction. He recovered from this episode with no noticeable deficits or sequelae. He remained on mechanical ventilation and developed endotracheal tube leakage, which required multiple exchanges; tracheomalacia and stenosis were suspected on CT imaging. He was monitored for repeated leaks despite doing well on volume support ventilation and having minimal oxygen support needs.

A tracheostomy was performed on day 15 of the hospital course due to repeated failure of extubation. Following the procedure, the patient was unable to maintain positive pressure ventilation and tracheal occlusion with maximal cuff inflation. He also developed active aspiration at which point the tracheostomy tube was removed and exchanged for a standard endotracheal tube to protect his airway. Upon further inspection with bronchoscopy, the surgical team discovered a tracheal defect, which was confirmed to be a TEF at the level of C7/T1 on CT scan. The timing of fistulization was uncertain, but this discovery provided compelling evidence for the recurrent aspiration events and significant difficulty in airway management. Definitive surgical correction of the fistula was planned with a multimodal approach between otolaryngology, cardiothoracic surgery, and plastic and reconstructive surgery teams. Because of the patient's hemodynamic status and the difficult location of his fistula, repair was ultimately deferred until his overall condition and nutritional status was optimized. Percutaneous endoscopic gastrostomy and tracheostomy revision were performed on day 25 of his hospital course. After medical and nutritional optimization, plans were made for surgical repair of the TEF with a transverse cervical flap coverage on day 40 of hospitalization.

\section{Surgical Technique}

The case was performed in conjunction with the otolaryngology head and neck surgery and cardiothoracic surgery teams. A median sternotomy and left cervical incision were deemed to be the best approach to yield the greatest exposure of the TEF. The TEF was identified 4 to $5 \mathrm{~cm}$ proximally to the carina and was approximately $1 \times 1.5 \mathrm{~cm}$ (-Fig. 1.) Primary closure of both the esophagus and the trachea was 


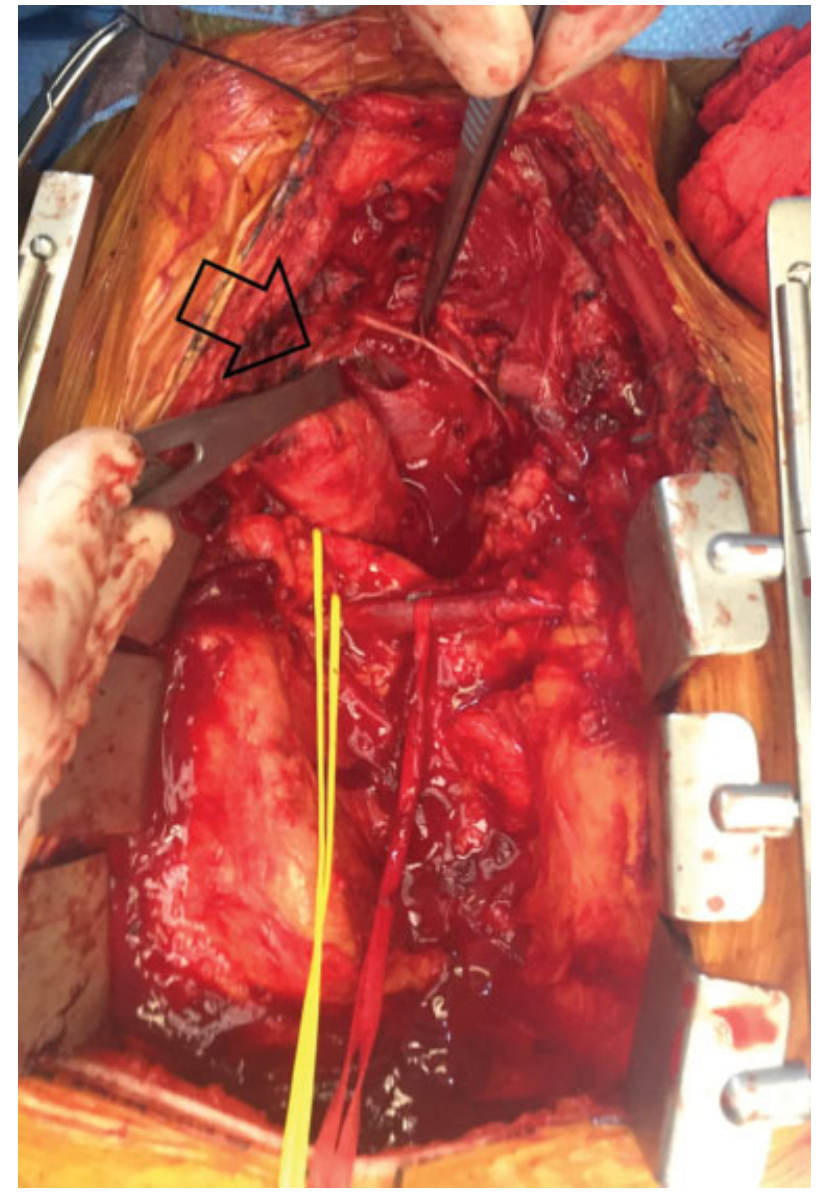

Fig. 1 Intraoperative photo showing tracheoesophageal fistula (arrowhead).

performed by CT surgery, which left a $3 \mathrm{~cm}$ semitransverse suture line on the anterior wall of the esophagus and on the posterior wall of the trachea. Due to the proximity of the defect and the relative consistency of the anatomy, a left transverse cervical flap was chosen for reconstruction.

The left transverse cervical artery (TCA) was located using a pencil Doppler and found to be $3 \mathrm{~cm}$ from midline and $2 \mathrm{~cm}$ cephalad to the clavicle at the anterior border of the sternocleidomastoid as described by Tessler et al. ${ }^{20}$ The artery was then followed $15 \mathrm{~cm}$ on its posterior branch beyond the superior edge of the trapezius muscle. A flap measuring $15 \mathrm{~cm} \times 4 \mathrm{~cm}$ was designed along the course of the TCA (-Fig. 2.) The main pedicle was dissected and elevated in a medial to lateral and lateral to medial combined approach (-Fig. 3). During elevation, the greater auricular and spinal accessory nerves were identified and protected.

After ensuring proper reach of the pedicle, the posterior skin flap was de-epithelialized and tunneled under the left sternocleidomastoid muscle ( - Fig. 4). The flap was then inset over the esophageal repair ensuring more than adequate coverage was achieved ( $\mathbf{- F i g . 5}$ ). The flap was also secured to the edge of the tracheal repair ( - Fig. 6). After the integrity of the repair and the vascularity of the flap were deemed satisfactory, a no. 0 Jackson Pratt drain was placed at the distal aspect of the repair and a no. 15 Blake drain was placed at the

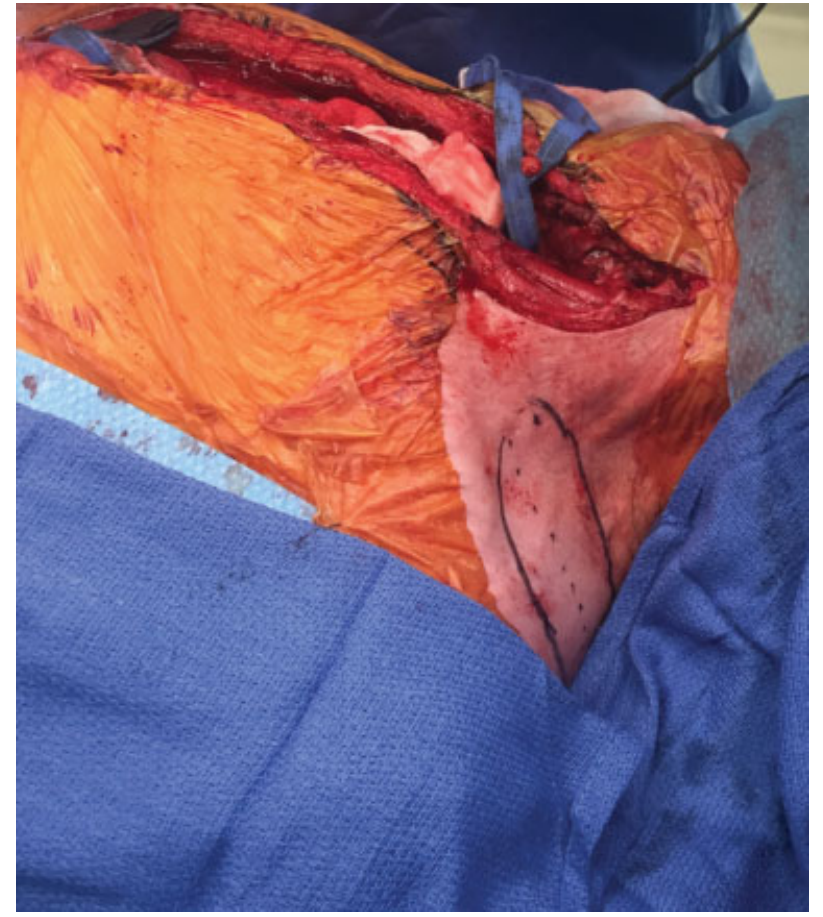

Fig. 2 Intraoperative photo portraying the transverse cervical artery flap.

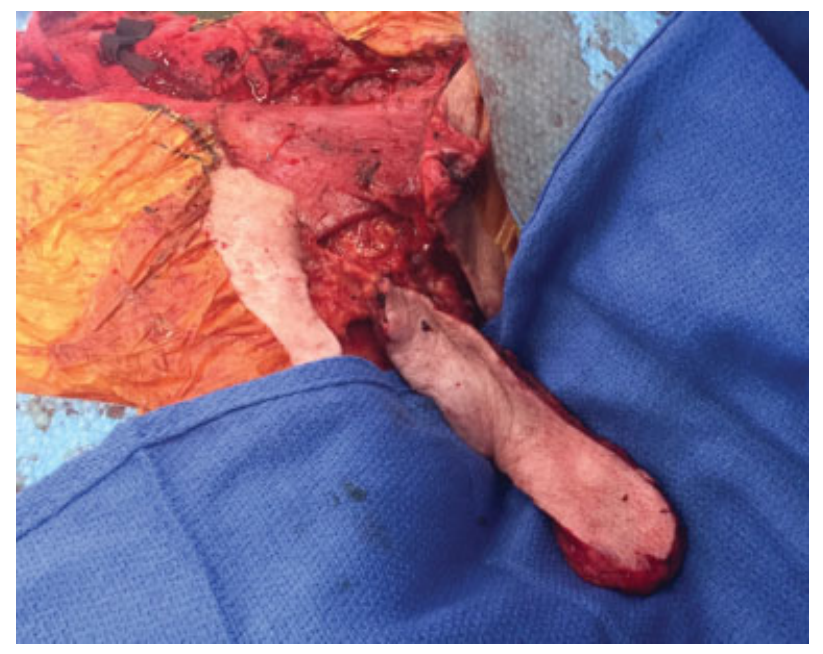

Fig. 3 Dissected and elevated transverse cervical artery flap.

left supraclavicular donor site area. At this point, care of the patient was turned back over to CT surgery for closure of the mediastinum and sternum, while the plastic surgery team continued to close the left supraclavicular donor site.

\section{Postoperative Course}

The repair went smoothly with no complications, and the patient was immediately transferred back to the intensive care unit. He was extubated on postoperative day (POD) 1 following a successful breathing trial; however, he was reintubated on POD 3 due to hypercapnic respiratory failure secondary to his limited capacity to clear secretions and global respiratory weakness. Otherwise, the repair remained viable with no complications which was confirmed 


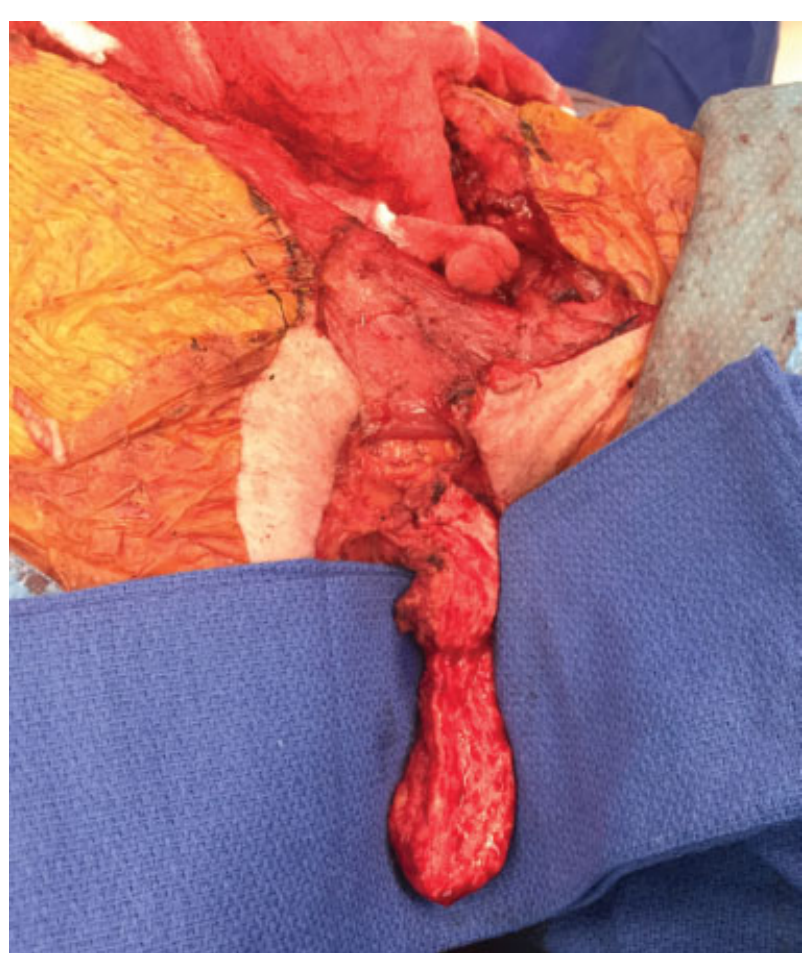

Fig. 4 Intraoperative photo of de-epithelialized flap.

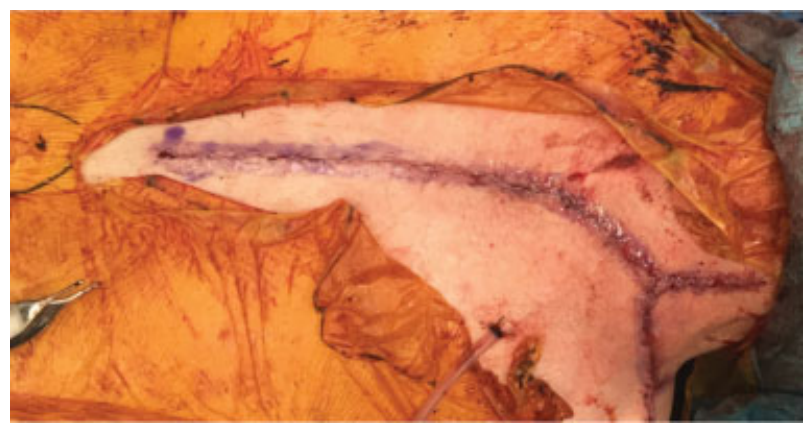

Fig. 5 Intraoperative photo showing final reconstruction.

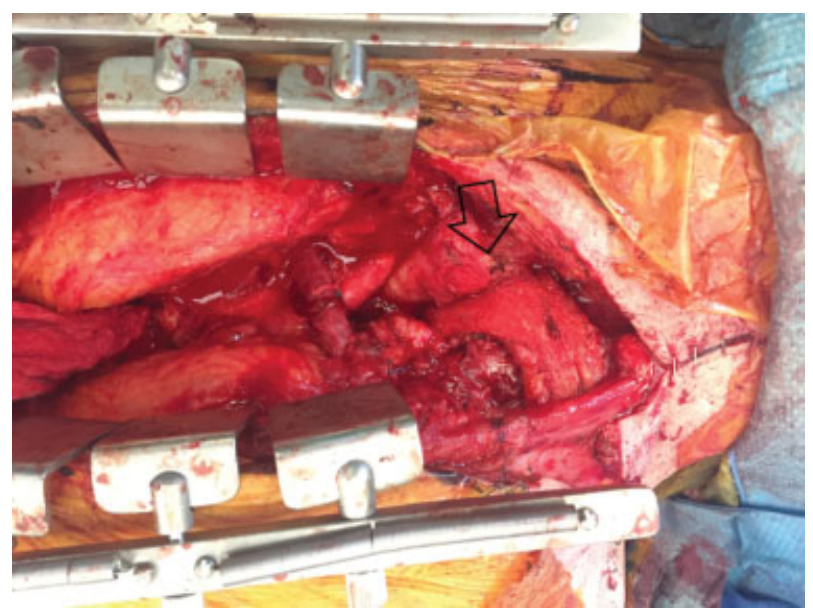

Fig. 6 Intraoperative photo showing inset (arrowhead) of the transverse cervical artery flap. under bronchoscopic exam. A tracheotomy was performed on POD 14. The procedure was tolerated well with no identifiable issues and minimal vent settings. The patient progressed favorably and was evaluated with a follow-up esophagram, swallow study, and bronchoscopy which showed the repair had maintained its integrity with no leaks. His clinical course improved until the patient was medically stable and discharged to a long-term, acute care hospital for rehabilitation, and is currently doing well.

\section{Discussion}

Because spontaneous closure rarely occurs, surgical intervention is typically required for effective and definitive repair of a TEF. Interventional techniques and adjunct supportive therapy may serve as temporizing measures to stabilize patients and optimize them for surgical repair to address potential leaks. ${ }^{12}$ Interventional techniques include esophageal stenting, airway stenting, or a combination of both. ${ }^{2}$ In addition, endoscopic closure using a variety of other methods has been reported, but with limited success, cardiac septal defect occluders, silicon rings, or vascular plugs have been reported to close the fistula. ${ }^{13,14}$ Like interventional management, a plethora of approaches have been used to surgically correct acquired TEF; however, repair remains difficult, with complication rates reported up to $55 \%$ in large retrospective studies. ${ }^{9,11}$ The most common approaches include initial cervical approach or cervical approach combined with a sternotomy for access, followed by tracheoesophageal repair.

Meticulous surgical repair of both tracheal and esophageal defects is essential due to the complexity of operating on an airway while maintaining ventilation and the propensity for complications, which themselves are related to the physiology of both the airway and the muscular tract of the esophagus. Normal functions, such as air movement and peristalsis, make healing a mechanical and dynamic process that can jeopardize the integrity of repair. Ventilation can be managed in different ways, including manual oxygen jet ventilation, high-frequency jet ventilation, distal tracheal intubation, spontaneous ventilation, and cardiopulmonary bypass. ${ }^{15}$ Final repair decisions are ultimately determined by the etiology of TEF; thus, the following techniques and usages are specific to benign postintubation fistulization.

Tracheal repair management poses a complex undertaking, and defects are typically managed with concomitant tracheal resection and reconstruction (TRR) with end-to-end anastomosis. Depending on the level of involvement, resection and reconstruction may also involve laryngeal anatomy (LTRR). Furthermore, larger defects may be repaired using TRR or LTRR over a tracheal T-tube. ${ }^{11,15-19}$ To ease resection and anastomosis, tracheal mobilization and release procedures may be utilized to allow for larger resection and less tension on the anastomosis, which is the primary cause of post-repair complications and morbidity. Thus, the anatomic level of fistulization necessitates special consideration in regard to the surgical approach. ${ }^{15,18,20}$

Esophageal repair typically consists of two-layer closure, one-layer closure, esophagostomy, or end-to-end esophageal 
anastomosis. Of note, esophagostomy is typically adjunct therapy, and end-to-end anastomosis is usually reserved as a last resort for repair. Two-layer closure is the most significant and widely used technique due to both the structural integrity and long-term viability of repair. This is due to higher bursting wall tension and superior healing compared with other techniques; however, closure involves specific directional placement of sutures in the mucosal layer followed by the muscular layer of the esophagus. The directionality of the sutures ensures balance of the forces generated by physiologic muscular contractions and helps prevent dehiscence of the repair. ${ }^{12,16,19-21}$ The esophageal and tracheal repairs are then buttressed together using alloderm, intercostal muscle flaps, omental flaps, or other free flaps. ${ }^{12,17,18,22}$ Our case offers a novel surgical approach using a pedicled TCA flap to buttress the tracheal and esophageal closures in the repair of a nonmalignant acquired TEF. Tessler et al describe the TCA flap as being a reliable pedicle with a reproducible and easy dissection. ${ }^{23}$ Due to the location of the TEF and the robust pedicle of TCA, the TCA pedicled flap was deliberately chosen to reinforce the esophageal and tracheal repair. Currently, there are no reported cases using this technique, yet this may offer a stronger surgical option given the high complication rates with currently used flaps. Use of the TCA flap may also offer further advantages such as low donor site morbidity, ease of harvest, and minimal resulting functional or aesthetic deficit. ${ }^{23}$ In addition, it is advantageous to interventional stenting techniques as it definitely closes the TEF.

In conclusion, definitive surgical repair of acquired TEF has a high reported complication rate. Although the TCA flap is described in the literature for use in head and neck oncologic reconstruction, we propose the TCA flap as a viable technical option to reinforce the surgical esophageal and tracheal repair. It is a reliable flap with a robust and lengthy pedicle that is in close proximity to the defect and has low donor site morbidity

\section{Conflict of Interest}

None declared.

\section{References}

1 Mooty RC, Rath P, Self M, Dunn E, Mangram A. Review of tracheoesophageal fistula associated with endotracheal intubation. J Surg Educ 2007;64(04):237-240

2 Ke M, Wu X, Zeng J. The treatment strategy for tracheoesophageal fistula. J Thorac Dis 2015;7(4, Suppl 4)S389-S397

3 Payne DK, Anderson WM, Romero MD, Wissing DR, Fowler M. Tracheoesophageal fistula formation in intubated patients. Risk factors and treatment with high-frequency jet ventilation. Chest 1990;98(01):161-164

4 Ji F, Nie P, Yi F, Zhang L. Management of esophageal stentingassociated esophagotracheal fistula, tracheal stenosis and tra- cheal rupture: a case report and review of the literature. Int J Clin Exp Pathol 2015;8(08):9332-9336

5 Hammoudeh ZS, Gursel E, Baciewicz FA Jr. Split latissimus dorsi muscle flap repair of acquired, nonmalignant, intrathoracic tracheoesophageal and bronchoesophageal fistulas. Heart Lung Circ 2015;24(06):e75-e78

6 Aggarwal D, Mohapatra PR, Malhotra B. Acquired Bronchoesophageal Fistula. Lung India 2009;26(01):24-25

7 Harley HR. Ulcerative tracheo-oesophageal fistula during treatment by tracheostomy and intermittent positive pressure ventilation. Thorax 1972;27(03):338-352

8 Collier KP, Zubarik RS, Lewis JH. Tracheoesophageal fistula from an indwelling endotracheal tube balloon: a report of two cases and review. Gastrointest Endosc 2000;51(02):231-234

9 Reed MF, Mathisen DJ. Tracheoesophageal fistula. Chest Surg Clin N Am 2003;13(02):271-289

10 Bibas BJ, Guerreiro Cardoso PF, Minamoto H, et al. Surgical Management of Benign Acquired Tracheoesophageal Fistulas: A Ten-Year Experience. Ann Thorac Surg 2016;102(04):1081-1087

11 Pelc P, Prigogine T, Bisschop P, Jortay A. Tracheoesophageal fistula: case report and review of literature. Acta Otorhinolaryngol Belg 2001;55(04):273-278

12 Muniappan A, Wain JC, Wright CD, et al. Surgical treatment of nonmalignant tracheoesophageal fistula: a thirty-five year experience. Ann Thorac Surg 2013;95(04):1141-1146

13 Pinsonneault C, Fortier J, Donati F. Tracheal resection and reconstruction. Can J Anaesth 1999;46(5 Pt 1):439-455

14 Li J, Gao X, Chen J, Lao M, Wang S, Zeng G. Endoscopic closure of acquired oesophagorespiratory fistulas with cardiac septal defect occluders or vascular plugs. Respir Med 2015;109(08):1069-1078

15 Barker J, Martino R, Reichardt B, Hickey EJ, Ralph-Edwards A. Incidence and impact of dysphagia in patients receiving prolonged endotracheal intubation after cardiac surgery. Can J Surg 2009;52(02):119-124

16 Auchincloss HG, Wright CD. Complications after tracheal resection and reconstruction: prevention and treatment. J Thorac Dis 2016;8(2, Suppl 2)S160-S167

17 Couraud L, Ballester MJ, Delaisement C. Acquired tracheoesophageal fistula and its management. Semin Thorac Cardiovasc Surg 1996;8(04):392-399

18 Luca N, Santana MJ, Festa BM, Collurà F, Righini S. Transverse Cervical Artery Perforator Flap: Standardized Surgical Technique and Multiple Reconstructive Opportunity in Head and Neck Surgery. Ann Plast Surg 2017;79(06):577-582

19 Di Carlo I, Toro A, Burrafato G, Gayet B. Repair of esophageal perforation: a new technique. Can J Surg 2008;51(03):E71-E72

20 Tessler O, Gilardino MS, Bartow MJ, et al. Transverse Cervical Artery: Consistent Anatomical Landmarks and Clinical Experience with Its Use as a Recipient Artery in Complex Head and Neck Reconstruction. Plast Reconstr Surg 2017;139(03):745e-751e

21 Oakes MG, Hosgood G, Snider TG III, Hedlund CS, Crawford MP. Esophagotomy closure in the dog. A comparison of a double-layer appositional and two single-layer appositional techniques. Vet Surg 1993;22(06):451-456

22 Erdim I, Sirin AA, Baykal B, Oghan F, Guvey A, Kayhan FT. Treatment of large persistent tracheoesophageal peristomal fistulas using silicon rings. Rev Bras Otorrinolaringol (Engl Ed) 2017; 83(05):536-540

23 Dartevelle P, Macchiarini P. Management of acquired tracheoesophageal fistula. Chest Surg Clin N Am 1996;6(04):819-836 